\title{
An optic disc grid: its evaluation in reproducibility studies on the cup/disc ratio
}

\author{
R. A. HitChings, C. GENIO, S. ANDERTON, AND P. CLARK* \\ From the Glaucoma Unit, Moorfields Eye Hospital, High Holborn, London WC1V 7AN
}

SUMMARY A grid system is described which may be superimposed upon stereoscopic pairs of optic disc photographs. This grid system allowed reproducible inter-and intraobserver measurements of the cup/disc (C/D) ratio. The surface contour of the optic disc was not clear-cut in approximately $10 \%$ of the population studied, which led to a reduction in reproducibility of the measurements made within the group as a whole. As a result it was found that a large increase in the $\mathrm{C} / \mathrm{D}$ ratio would have to occur for such change to be of statistical significance. Even with an optic grid as a reference system the serial measurements of the $C / D$ ratio are unlikely to be of value in the management of patients with chronic glaucoma.

The importance of making accurate measurements of the optic disc in the management of chronic glaucoma has increased following 2 observations. Firstly, the presence of visual field defects may be suggested by looking at the optic disc, ${ }^{12}$ and, secondly, visible changes at the optic disc may precede visual field loss. ${ }^{34}$ Visual field loss may be related to the vertical cup/disc (C/D) ratio, the chance of a visual field defect being present dramatically increasing if the $C / D$ ratio exceeds $0 \cdot 7 .^{5}$ However, $\mathrm{C} / \mathrm{D}$ ratios are usually measured by eye. The number written in the patients records suggests a precision which has not been based on repeated inter- or intraobserver observation. ${ }^{6-8}$ In consequence a number of reference or grid systems have been developed which provide reference points for more reproducible measurements. ${ }^{9}$

This paper describes a simple and easy-to-use grid system for measuring the C/D ratio on disc photographs and assesses the results obtained on repeated measurements by 3 observers. It further assesses the minimum change in $C / D$ ratio required to fall outside intraobserver variation.

\section{Material and methods}

OPTIC DISC GRID

Two grids, mirror images, were devised. Each grid consisted of a series of 6 lines radiating away from a central point. Four of these lines met in the form of a cross; the other 2 were found either to the right or to Correspondence to Mr R. A. Hitchings.

${ }^{*}$ Statistician, Institute of Ophthalmology, Judd Street, London WC1. the left of the vertical meridian bisecting the rightangles of this cross (Fig. 1). This allowed measurements in the right and left temporal halves of the optic disc in the right and left eye.

A circle, chosen in size to agree with the average disc diameter seen in photographs taken with the West German Zeiss Fundus Camera, was centered on the meeting point of these 6 lines. Each line was subdivided, the 5th subdivision corresponding with the circumference of the circle. The grid was mounted

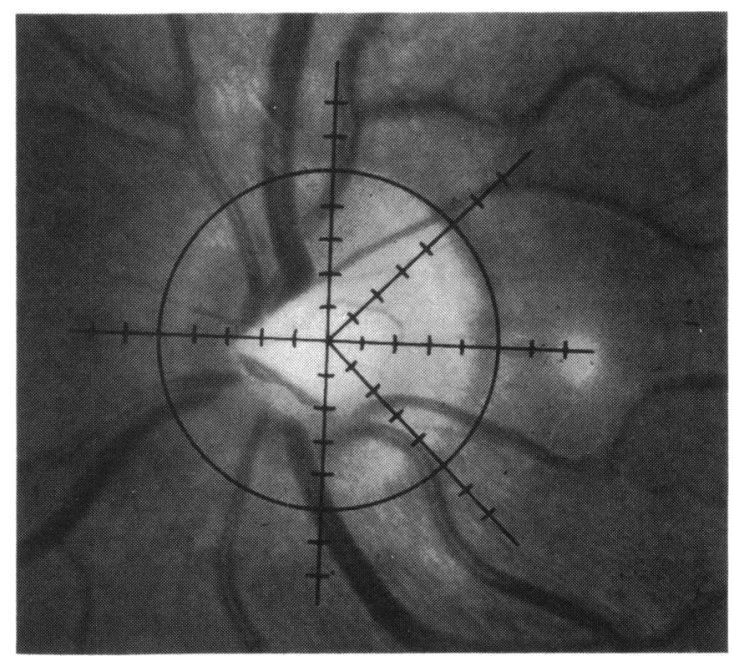

Fig. 1 Optic disc grid superimposed on black-and-white photograph of the optic disc. 


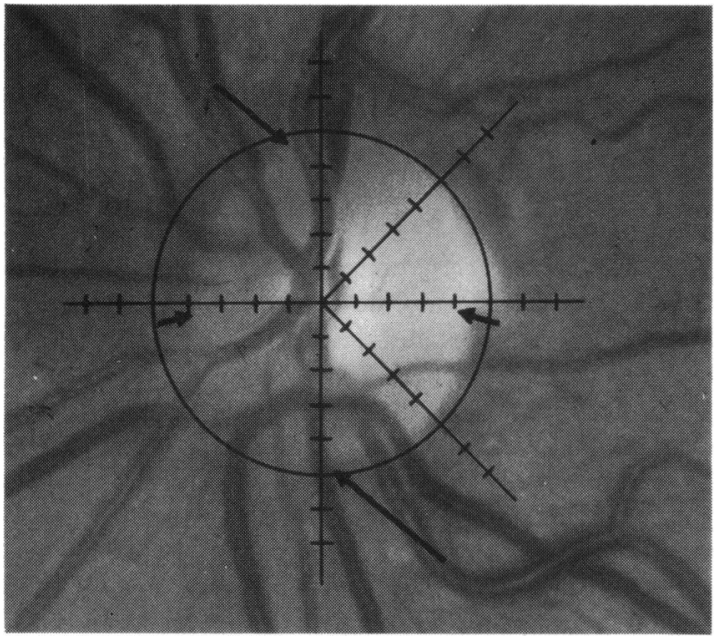

Fig. 2 Centring of a symmetrically oval disc. The horizontal diameter (short arrows) is less than the vertical diameter (long arrows).

on transparent plastic which could then be superimposed on an optic disc photograph (Fig. 1).

Centring of the grid on an optic disc was important. The majority of discs were found to be either circular or symmetrically oval with a long axis and a short axis. Centring of a circular disc was not a problem. Centring of a symmetrically oval disc was carried out so that the centre of the grid circle was placed at the point where the line running through the long axis bisected the line running through the short axis (Fig. 2).

Centring of optic discs with an irregular outline was

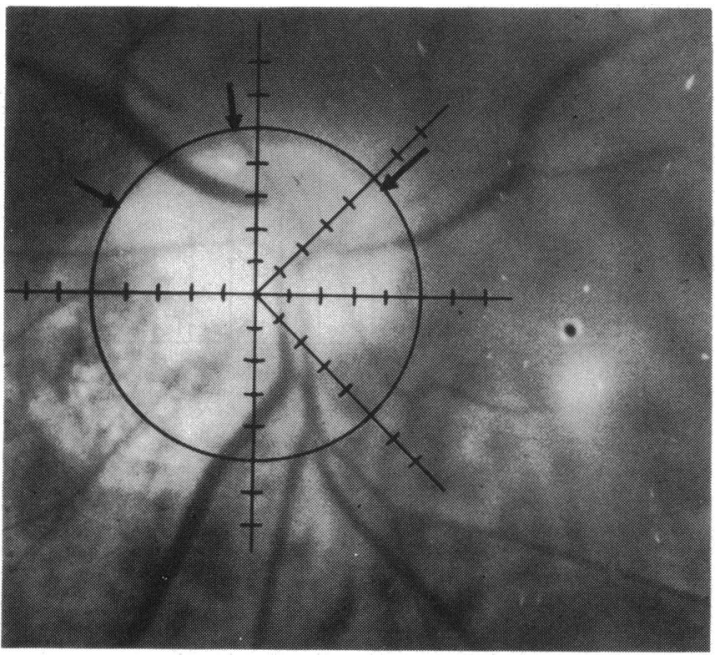

Fig. 3 Centring of a grossly asymmetric disc showing a best fit of that part of the disc circumference which corresponds through the circle of the grid (arrows). carried out by a process of 'completing the circle.' The part of the disc rim which formed an arc of the circle was fitted to the rim of the grid circle (Fig. 3). An imaginary line was drawn to complete this circle, the whole having the same radius as the arc formed by the 'fitted' part of the disc rim. Finally the horizontal line of the grid was aligned to bisect the fovea, ensuring that no variation from rotation of the grid on the disc had occurred.

Measurements were taken along each radius to the nearest half division-for example, $3,3.5$, or 4 divisions. Measurements were made from the 'disc centre' to both the disc margin and the cup margin. The $C / D$ ratio for each radius could then be expressed as a fraction. For the purpose of this study, to allow comparison with previous studies, this fraction was converted into 10ths.

\section{DIMENSIONS OF THE OPTIC DISC}

The demarcation between cup and rim was called the cup orifice. Measurement along the orifice provided figures for the $\mathrm{C} / \mathrm{D}$ ratio. The photographs were not stereo pairs, for they were photographed with a variable stereo base. ${ }^{2}$ The ease of visualisation of the cup orifice increased with both magnification and increasing the 'stereo effect' by adjusting the stereo base. To identify the demarcation between neuroretinal rim and wall of the optic cup an imaginary straight line was drawn along the surface of the inner retina crossing the optic disc. This line provided reference points for the height of the neuroretinal rim. That part of the optic disc which was in contact with this line was considered to be neuroretinal rim,

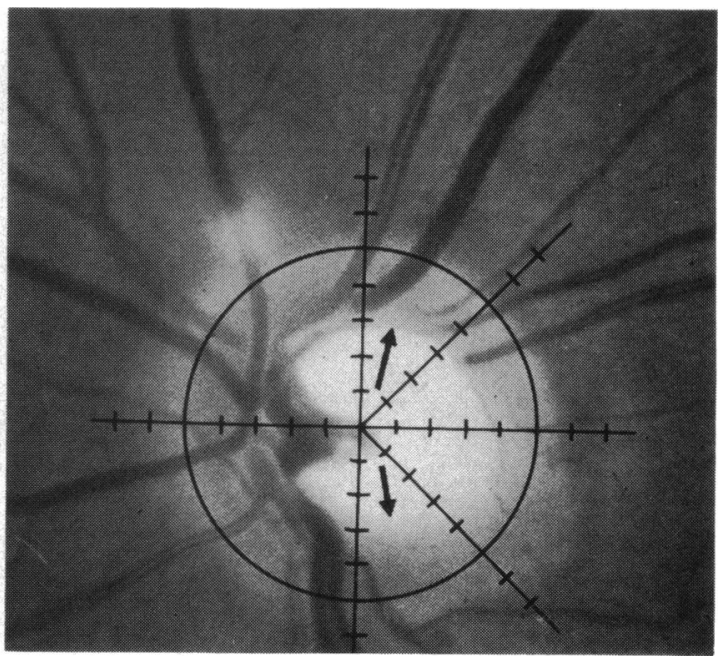

Fig. 4 Sharply outlined neuroretinal rim-cup boundary allowing ease of measuring cup/disc ratios (arrows). 


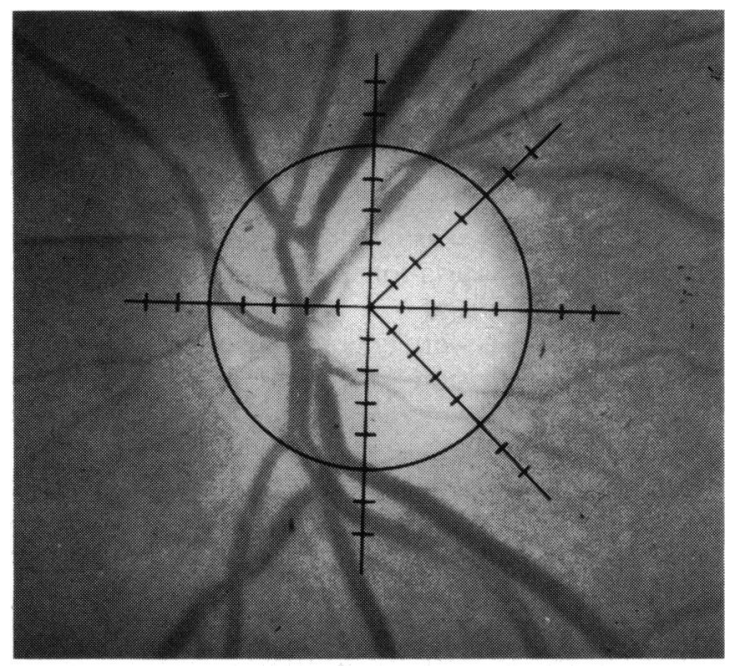

Fig. 5 Ill-defined neuroretinal rim to cup boundary. This lack of definition was found to be the source of major errors in repeated measurements.

while that part of the optic disc which dipped below this imaginary line was the optic cup. The orifice of the optic cup was considered to extend up to this imaginary line. For those eyes with a vertically sided or 'undercut' optic cup it was easy to estimate the position of the cup orifice (Fig. 4). Many eyes, however, had a shelving optic cup, at least in part (Fig. 5). It was for these eyes that this arbitrary definition of the neuroretinal rim and cup orifice proved invaluable. Care was taken to differentiate between the edge of the neuroretinal rim and adjacent peripapillary halo

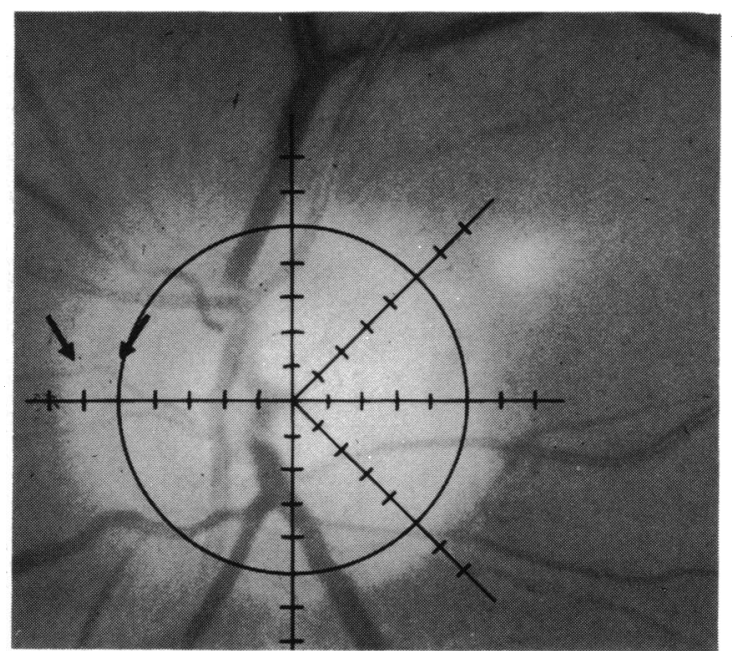

Fig. 6 Peripapillary halo (arrows) surrounding the optic disc which, in this instance, has the same diameter at the circle of the grid.
(Fig. 6). A halo was considered to be present if a pale zone was seen round all or part of the edge of the optic disc. The dividing line between the edge of the optic disc and this pale halo was sometimes marked by pigment and always by a colour change.

\section{PROCEDURE}

Sixty stereo pairs were chosen from the ocular hypertensive patients on the file of the Glaucoma Unit at Moorfields Eye Hospital, High Holborn. The photographs were obtained by a method previously described. ${ }^{2}$ Each stereo pair was chosen so that it provided a good stereo effect and the surface of the optic disc was in good focus. Each photograph was taken under standard magnification (as opposed to being photographed with the $\times 2$ adaptor).

Each observer examined each photograph on 3 separate occasions. The examination was carried out in a masked fashion each time. The rules for measuring the $C / D$ ratio outlined above were followed. These figures allowed the determination of inter- and intraobserver variability. The first set of results obtained by observer no. 2 were converted into figures for the overall vertical and horizontal C/D ratio.

\section{Results}

From the first set of results obtained by observer no. 2 an idea of the study population may be obtained. The prevalence of each C/D ratio has been noted and set out in Fig. 7.

Table 1 sets out the mean C/D ratio for radii 1-6 for each of the 3 observers. A note is also made of the standard deviation.
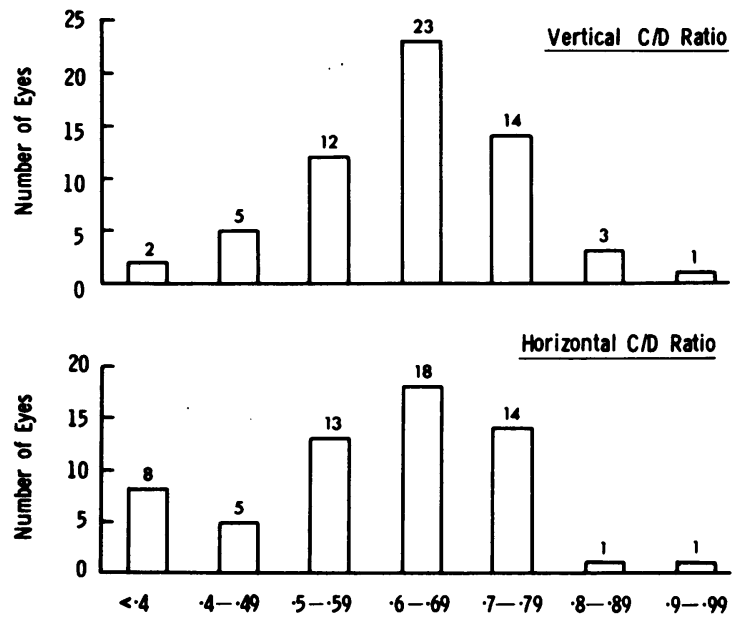

Fig. 7 Histograms to show the prevalence of different $C / D$ ratios in the cases studied. 
Table 1 Mean $C / D$ ratio for radii 1-6 for observers

\begin{tabular}{lllllll}
\hline \multirow{2}{*}{ Observer } & \multicolumn{4}{l}{ Radii (mean value and $S D$ ) } \\
\cline { 2 - 7 } & 1 & 2 & 3 & 4 & 5 & 6 \\
\hline 1 & $0 \cdot 65(0 \cdot 12)$ & $0 \cdot 65(0 \cdot 145)$ & $0 \cdot 63(0 \cdot 165)$ & $0 \cdot 60(0 \cdot 165)$ & $0 \cdot 59(0 \cdot 165)$ & $0 \cdot 58(0 \cdot 165)$ \\
2 & $0 \cdot 675(0 \cdot 135)$ & $0 \cdot 77(0 \cdot 148)$ & $0 \cdot 695(0 \cdot 142)$ & $0 \cdot 66(0 \cdot 165)$ & $0 \cdot 61(0 \cdot 20)$ & $0 \cdot 60(0 \cdot 215)$ \\
3 & $0 \cdot 61(0 \cdot 16)$ & $0 \cdot 60(0 \cdot 165)$ & $0 \cdot 59(0 \cdot 145)$ & $0 \cdot 575(0 \cdot 142)$ & $0 \cdot 56(0 \cdot 152)$ & $0 \cdot 58(0 \cdot 165)$ \\
\hline
\end{tabular}

Table 2 Minimum significant change in $C / D$ ratio for radii $1-6(p<0.05)$ based on 3 readings

\begin{tabular}{lllllll}
\hline \multirow{2}{*}{ Observer } & \multicolumn{3}{l}{ Radii } & & & \\
\cline { 2 - 6 } & 1 & 2 & 3 & 4 & 5 & 6 \\
\hline 1 & $0 \cdot 18$ & $0 \cdot 19$ & $0 \cdot 15$ & $0 \cdot 22$ & $0 \cdot 23$ & $0 \cdot 26$ \\
2 & $0 \cdot 20$ & $0 \cdot 29$ & $0 \cdot 31$ & $0 \cdot 24$ & $0 \cdot 33$ & $0 \cdot 29$ \\
3 & $0 \cdot 17$ & $0 \cdot 21$ & $0 \cdot 28$ & $0 \cdot 22$ & $0 \cdot 25$ & $0 \cdot 24$ \\
\hline
\end{tabular}

In clinical practice it is important to know what is the minimum increase in $\mathrm{C} / \mathrm{D}$ ratio that has to occur for this observed change to be outside the variation of previous observations. A statistical method was derived (see Appendix) to show the minimum statistically significant change in C/D ratio. (It should be remembered that this exercise was carried out on repeated examinations of the same photograph. In clinical practice other errors from sequential photography would occur that could increase the size of the minimum statistically significant change.) These values have been set out in Table 2 .

Table 3 sets out the maximum difference between 3 readings to give a worst figure for intraobserver variations. The figures show the mean and the standard deviation of the sum of these maximum differences. A note is also made of the number of eyes for radii 1-6 where the maximum difference between the readings exceeded $0 \cdot 2$.

Table 4 notes the figures for interobserver variation. The mean and the standard deviation of the sum of the maximum differences recorded at the time of the third of the 3 readings by each observer have been found. A note is also made of the number of eyes where the maximum difference between the 3 observers for the individual $C / D$ ratios exceeded $0 \cdot 2$ and $0 \cdot 4$.
Table 5 sets out the number of eyes where the 3 observers agreed on the $C / D$ ratio within $1 / 10$ th.

\section{Discussion}

This discussion will briefly look at previous grid systems, the value of using a superimposed grid when examining the optic disc, and the accuracy of measurements obtained with the grid described in this paper.

\section{PREVIOUS GRID SYSTEMS}

Hollows and McGuiness ${ }^{10}$ produced a series of different sized circles to identify differing cup sizes. Their system did not allow for eccentricity or noncircularity of the optic cup and concentrated on the cup rather than changes occurring at the neuroretinal rim. Shaffer et al. ${ }^{11}$ introduced a 10 -square diameter grid which was superimposed upon optic disc photographs allowing dimensions of the central cup to be noted. Gloster and Parry 12 assessed dimensions of the disc and cup by projecting disc photographs on to a sheet of paper and drawing a line around the orifice of the optic cup. Gloster ${ }^{5}$ later demonstrated the use of stereo photographs to identify the cup orifice and then, from examination of the optic disc with Hruby lens examination, drew a line to mark this orifice on a Polaroid black-and-white photograph of the optic disc that had been taken at the same time. Measurements were taken from this line. Shiose ${ }^{13}$ employed simultaneous projection of an optic disc photograph and a superimposed grid on to a screen. Although this grid concentrated on changes occurring at the neuroretinal rim, both at the upper and lower poles, to identify eyes with visual field loss from glaucoma, his system concentrated on colour changes rather than topographical ones.

Table 3 Intraobserver variations: maximum difference between readings

\begin{tabular}{|c|c|c|c|c|c|c|}
\hline & \multicolumn{6}{|l|}{ Radii } \\
\hline & 1 & 2 & 3 & 4 & 5 & 6 \\
\hline $\begin{array}{l}\text { Mean (SD) maximum } \\
\text { differences } \\
\text { No. of eyes with maximum }\end{array}$ & $0.09(0 \cdot 11)$ & $0 \cdot 10(0 \cdot 17)$ & $0 \cdot 12(0 \cdot 18)$ & $0 \cdot 11(0 \cdot 13)$ & $0 \cdot 15(0 \cdot 17)$ & $0 \cdot 14(0 \cdot 15)$ \\
\hline difference $>0 \cdot 20$ & 4 & 6 & 6 & 6 & 17 & 15 \\
\hline
\end{tabular}


Table 4 Interobserver variation. The means of the maximum differences recorded in $C / D$ ratio for each of the 102 stereo photographs together with a standard deviation have been noted for radii 1-6

\begin{tabular}{|c|c|c|c|c|c|c|}
\hline & \multicolumn{6}{|l|}{ Radii } \\
\hline & 1 & 2 & 3 & 4 & 5 & 6 \\
\hline $\begin{array}{l}\text { Mean }(\mathrm{SD}) \text { of the third } \\
\text { reading }\end{array}$ & $0 \cdot 15(0 \cdot 10)$ & $0 \cdot 21(0 \cdot 20)$ & $0 \cdot 20(0 \cdot 18)$ & $0 \cdot 20(0 \cdot 14)$ & $0 \cdot 21(0 \cdot 16)$ & $0.19(0.16)$ \\
\hline readings $>0.2$ & 12 & 21 & 20 & 21 & 24 & 27 \\
\hline$>0.4$ & 2 & 4 & 1 & 6 & 5 & 3 \\
\hline
\end{tabular}

Table 5 Number of eyes in which 3 observers agreed on $C / D$ ratio to within $1 / 10 C / D$ ratio

\begin{tabular}{lllllll}
\hline & \multicolumn{3}{l}{ Radii } & & & \\
\cline { 2 - 7 } & 1 & 2 & 3 & 4 & 5 & 6 \\
\hline No. of eyes & 52 & 55 & 51 & 52 & 52 & 48 \\
\hline
\end{tabular}

The optic disc grid described in this paper was chosen for simplicity, for it allows superimposition of the transparent grid on a stereo photograph of the optic disc. This system was found to be simple to use and easy to teach to paramedical personnel, while the grid itself was cheap to produce. Topography rather than colour change was chosen because of previous studies emphasising the importance of the size of the cup orifice and thickness in the neuroretinal rim when assessing changes at the optic disc seen in glaucoma. ${ }^{125}$

\section{VALUE OF MEASUREMENTS MADE WITH THIS SUPERIM POSED GRID}

Subdivision of horizontal and vertical diameters into radii allowed the distinction to be made between generalised enlargement of the cup and focal extension. Radii 2 and 4 (Fig. 1) allowed identification of focal changes in the upper and lower temporal part of the neuroretinal rim, that is, those parts of the neuroretinal rim corresponding with the arcuate fibre region in the visual field. The use of 6 radii provided a good picture of the overall dimensions of the orifice of the optic cup.

It may be seen from Fig. 1 that the study population consisted in the main of eyes with a vertical C/D ratio ranging between 0.5 and $0 \cdot 7$. A review of these photographs at the conclusion of the study showed that a fair proportion of them did not have clearly demarcated edges to the junction between optic cup and neuroretinal rim. No patient, however, had areas of pallor or absence of the neuroretinal rim that would be expected in patients with visual field defects. ${ }^{2}$

Examination of Table 1 and Table 3 gives figures for intraobserver variations. It may be seen from
Table 1 that radii $1,3,4,5$, and 6 had differences in the mean value obtained by each of the 3 observers to be at or below 1/10th of a disc diameter. From examination of Table 3 the maximum difference between the C/D ratio averaged out at $<0.12$ in 4 out of the 6 radii. The least accurate radius (no. 5) was that drawn to the 6 o'clock position in the optic disc. It appeared that for the study reported here the greatest number of sloping walls to the optic cup were found at this particular radius, and this was a major source of error. It should be seen that out of the 60 eyes studied for radii 5 and 6 the maximum difference on 3 readings $>0 \cdot 20$ was seen in 14 and 16 eyes respectively. The overall variation and particularly the standard deviations compared very well with the figures reported by Sommer et al. ${ }^{4}$

Table 2 shows the minimum significant increase in $\mathrm{C} / \mathrm{D}$ ratio for each of the 6 radii $(\mathrm{p}<0 \cdot 05)$. It will be seen that, although the 3 observers achieved apparently reproducible results, a statistically significant increase in the $C / D$ ratio for the 6 radii ranged between $0 \cdot 19$ and $0 \cdot 28$. This minimum increase in the $C / D$ ratio required before it can be said that the apparent change is not due to measurement error casts doubt on the value of measuring C/D ratio, even with a grid, in the long-term follow-up of patients with glaucoma.

Tables 1,4 , and 5 outline the interobserver variation. As would be expected there was a greater maximum difference between the 3 observers than between the measurements made by one observer. Even so the mean of this maximum difference ranged between 0.15 and 0.18 for all 6 radii. The mean radii noted by observers 1,2 , and 3 are set out in Table 1 . Even with the averaging process required to produce these figures there is still a major difference between the means in some instances. The agreement achieved between the 3 observers is set out in Table 5 . In over 50 of the 60 eyes agreement to within 1/10th of the C/D ratio was seen by the 3 observers for 5 of the 6 radii. However, the enormous variation that was seen with some eyes clouded the result as a whole.

It will be remembered that the photographs chosen 
for this study were all in good focus, and as a result 'accurate' measurements could be made. Additional errors will be made when focusing is less accurate. Sequential photography may not have the same alignment or magnification.

\section{SOURCES OF ERROR}

A number of errors in the method exist. Firstly, readings were made to the nearest one-half division, and errors could occur in estimating a reading in this way. It can be seen that the 3 observers agreed on the $\mathrm{C} / \mathrm{D}$ ratio to within $1 / 10$ th of the disc diameter in over 50 observations. An improvement in the method of measurement would be to employ a greater number of divisions of a larger grid with a more magnified photograph.

Secondly, errors in centring will be likely to cause variation in the results achieved. Although care was taken to follow the plan outlined above to ensure correct centring, the greatest variability seemed to occur in eyes with 'asymmetrical discs.' The most important source of error was seen in eyes with sloping walls to the optic cup having a gradual change between wall and neuroretinal rim. Again, greater magnification could largely overcome this problem.

\section{CONCLUSION}

The results presented in this paper show that it is possible to achieve a fair degree of reproducibility on multiple examinations of stereo photographs of the optic disc; and a fair degree of similarity was achieved when comparing the results made by different individuals. This was especially true when the photographs had surface contours sufficiently well marked to allow confident measurements. In the study population $10 \%$ of the optic discs had insufficiently well marked surface contours, producing a large variation on repeated measurements. This $10 \%$ was sufficient in numbers to lower the overall accuracy. As a result it was found that a large change in the dimensions of the orifice of the optic cup had to occur for it to be of statistical significance. The size of this required change precludes the use of a superimposed grid for serial follow-up of patients with chronic glaucoma; it also casts doubt on the value of measurements of the
$\mathrm{C} / \mathrm{D}$ ratio made by eye in the long-term management of this disease.

\section{Appendix}

Calculation of significant differences in $\mathrm{C} / \mathrm{D}$ ratios.

(1) An estimate of within-subject variance of the measurements was calculated from the data.

(2) Any subsequent 'before' and 'after' measurements would then be regarded as 2 independent samples from a normally distributed population with that variance.

(3) For a particular significance level the size of a significant difference between the means of the 2 samples could then be calculated.

We thank Kay Mills for typing the manuscript.

\section{References}

1 Hoskins HD, Gelber EC. Optic disc topography and visual field defects in patients with increased intraocular pressure. Am J Ophthalmol 1975; 80: 284-290.

2 Hitchings RA, Spaeth GL. The optic disc in glaucoma. II. Correlation of the appearance of the optic disc with the visual field. Br J Ophthalmol 1977; 61: 107-13.

3 Pederson JE, Anderson DR. The mode of progressive disc cupping in ocular hypertension and glaucoma. Arch Ophthalmol 1980; 98: 490-5.

4 Sommer A, Pollack I, Maumanee AE. Optic disc parameters and onset of glaucomatous field loss. 1. Methods and progressive changes in disc morphology. Arch Ophthalmol 1979; 97: 1444-8.

5 Gloster J. Quantitative relationship between cupping of the optic disc and visual field loss in chronic simple glaucoma. $\mathrm{Br} J$ Ophthalmol 1978; 62: 665-9.

6 Hollows FC, Graham PA. Intraocular pressure, glaucoma, and glaucoma suspects in a defined population. $\mathrm{Br} J$ Ophthalmol 1966; 50: 570-86.

7 Lichter P. Cited by Kaiser-Kupfer MI. Thesis for the American Society of Ophthalmology. Clinical research methodology in ophthalmology. Reprinted in the Trans Am Soc Ophthalmol Otolaryngol 1980; 78: 896-946.

8 Schwartz JT. Methodologic differences and measurement of cupdisc ratio. Arch Ophthalmol 1976; 94: 1101-5.

9 Sommer A. Epidemiology and statistics for the ophthalmologist. New York: Oxford University Press, 1980: 35.

10 Hollows FC, McGuiness R. The size of the optic cup. Trans Ophthalmol Soc NZ 1966; 19: 33-8.

11 Shaffer RN, Ridgway WL, Brown R, Kramer SG. The use of diagrams to record changes in glaucomatous disks. $A m J$ Ophthalmol 1975; 80: 460-4.

12 Gloster J, Parry DG. Use of photographs for measuring cupping in the optic disc. Br J Ophthalmol 1974; 58: 850-62.

13 Shiose Y. 'Quantative disc pattern' as a new parameter for glaucoma screening. Glaucoma 1979 ; i: 41-9. 